\title{
AN EXAMPLE OF A HARMONIC MAP BETWEEN EUCLIDEAN BALLS
}

\author{
ANTONIOS D. MELAS
}

(Communicated by Peter Li)

\begin{abstract}
Here we construct a homeomorphism of the unit sphere in threedimensional Euclidean space to itself whose harmonic extension in the unit ball is not a diffeomorphism.
\end{abstract}

A theorem of Rado [R] states that if $\Omega \subseteq \mathbb{R}^{2}$ is a bounded convex domain with smooth boundary $\partial \Omega$ and if $\varphi: S^{1} \rightarrow \partial \Omega$ is a homeomorphism then its harmonic extension $u: D \rightarrow \Omega$, where $D$ is the unit disc, is a diffeomorphism.

Here we will show that this is false in the 3-dimensional case. In fact, we will construct a homeomorphism $\varphi: S^{2} \rightarrow S^{2}$ such that its harmonic extension $u: B^{3} \rightarrow B^{3}$, where $B^{3}$ is the open unit 3-ball, is not a diffeomorphism.

Of course, this leaves open the question of whether the harmonic extension of a homeomorphism $\varphi: S^{2} \rightarrow S^{2}$ is a homeomorphism.

Notation. $S^{2}$ denotes the 2-sphere of radius 1 in $\mathbb{R}^{3}$ and $B^{3}$ the open unit ball in $\mathbb{R}^{3}$. For a continuous function $f: S^{2} \rightarrow \mathbb{R}$ its harmonic extension is the unique continuous function $v: \bar{B}^{3} \rightarrow \mathbb{R}$ such that $\Delta v=0$ in $B^{3}$ and $v=f$ on $\partial B^{3}=S^{2}$. For a $C^{1}$ function $g: \mathbb{R}^{3} \rightarrow \mathbb{R}^{3}, J_{g}$ denotes its Jacobian matrix.

Theorem. There exists a homeomorphism $\varphi: S^{2} \rightarrow S^{2}$ whose harmonic extension $u: B^{3} \rightarrow B^{3}$ satisfies det $J_{u}(0)=0$, hence it is not a diffeomorphism.

Proof. Given a continuous function $f: S^{2} \rightarrow \mathbb{R}$, its harmonic extension $v: B^{3}$ $\rightarrow \mathbb{R}$ is given by

$$
v(x)=\frac{1-|x|^{2}}{4 \pi} \int_{S^{2}} \frac{f(y)}{|x-y|^{3}} d \sigma(y)
$$

for $x \in B^{3}$, where $d \sigma$ denotes the surface measure on $S^{2}$. Hence

$$
\nabla v(0)=\frac{3}{4 \pi} \int_{S^{2}} x f(x) d \sigma(x) .
$$

Now choose coordinate axes $x_{1}, x_{2}, x_{3}$ so that 0 is the center of $B^{3}$ and let $r, \theta$ denote polar coordinates on the $x_{1} x_{2}$-plane. Let $\delta>0$ be small enough so that if

$$
E=\left\{x \in S^{2}: x_{3} \in[-1+\delta, 0] \cup[1-\delta, 1]\right\}
$$

Received by the editors June 26, 1991.

1991 Mathematics Subject Classification. Primary 54C20, 31 B99. 
then

$$
\int_{E} x_{3} d \sigma(x)<0
$$

By (4) there exists $\eta>0$ sufficiently small so that if

$$
\begin{aligned}
F= & \left\{x \in S^{2}: x_{3} \in[-1+\delta, 0],|\theta| \geq \eta,|\pi-\theta| \geq \eta\right\} \\
& \cup\left\{x \in S^{2}: x_{3} \in[0,1-\delta],\left|\theta-\frac{\pi}{2}\right| \leq \eta,\left|\theta+\frac{\pi}{2}\right| \leq \eta\right\} \\
& \cup\left\{x \in S^{2}: x_{3} \in[1-\delta, 1]\right\}
\end{aligned}
$$

then

$$
\int_{F} x_{3} d \sigma(x)<0 .
$$

The interior of $F$ and $S^{2} \backslash F$ are simply connected domains in $S^{2}$ whose common boundary is a Jordan curve $\gamma: S^{1} \rightarrow S^{2}$. Moreover, $\gamma$ is symmetric with respect to the $x_{1} x_{3}$ - and $x_{2} x_{3}$-planes. Also $(0,0,1) \in \operatorname{int} F$ and $(0,0,-1) \in S^{2} \backslash F$. Therefore there exist homotopies

$$
C_{t}: S^{1} \rightarrow F \quad \text { and } \quad C_{-t}: S^{1} \rightarrow \overline{S^{2} \backslash F}, \quad \text { where } 0 \leq t \leq 1,
$$

having the following properties:

(i) $C_{0}=\gamma$;

(ii) $C_{t}, C_{-t}$ are Jordan curves and are symmetric with respect to the $x_{1} x_{3}-$ and $x_{2} x_{3}$-planes for $0 \leq t<1$;

(iii) $C_{1}\left(S^{1}\right)=\{(0,0,1)\}$ and $C_{-1}\left(S^{1}\right)=\{(0,0,-1)\}$;

(iv) $C_{t_{1}}\left(S^{1}\right) \cap C_{t_{2}}\left(S^{1}\right)=\varnothing$ if $-1 \leq t_{1}<t_{2} \leq 1$;

(v) $S^{2}=\bigcup_{-1 \leq t \leq 1} C_{t}$.

Then for any continuous strictly increasing $h:[-1,1] \rightarrow[-1,1]$ such that $h(-1)=-1$ and $h(1)=1$, let $\varphi_{h}: S^{2} \rightarrow[-1,1]$ be defined by

$$
\varphi_{h}(x)=h(t) \text { if } x \in C_{t},-1 \leq t \leq 1 .
$$

By (ii), (iii), (iv), and (v), $\varphi_{h}$ is well defined and continuous on $S^{2}$ and, moreover, it is even in the $x_{1}$ and $x_{2}$ variables. Hence

$$
\int_{S^{2}} x_{i} \varphi_{h}(x) d \sigma(x)=0 \text { for } i=1,2 \text {. }
$$

By (iii) there exists $0<\varepsilon_{1}<1$ such that $C_{t} \subseteq\left\{x \in S^{2}: x_{3}>\frac{1}{2}\right\}$ and $C_{-t} \subseteq\left\{x \in S^{2}: x_{3}<-\frac{1}{2}\right\}$ if $1-\varepsilon_{1} \leq t \leq 1$. Hence there exist $\varepsilon_{2}>0$ sufficiently small such that if $h_{0}:[-1,1] \rightarrow[-1,1]$ is defined by $h_{0}(-1)=-1$, $h_{0}\left(-1+\varepsilon_{1}\right)=-\varepsilon_{2}, h_{0}\left(1-\varepsilon_{1}\right)=\varepsilon_{2}, h_{0}(1)=1$, and $h_{0}$ is linear in the intervals determined by the points $-1,-1+\varepsilon_{1}, 1-\varepsilon_{1}, 1$, then

$$
\int_{S^{2}} x_{3} \varphi_{h_{0}}(x) d \sigma(x)>0 \text {. }
$$

By (6) there exist $0<\varepsilon_{3}<1$ and $\varepsilon_{4}>0$ sufficiently small such that if $h_{1}:[-1,1] \rightarrow[-1,1]$ is defined by $h_{1}(-1)=-1, h_{1}\left(-1+\varepsilon_{3}\right)=-\varepsilon_{4}, h_{1}(0)=$ $0, h_{1}\left(\varepsilon_{3}\right)=1-\varepsilon_{4}, h_{1}(1)=1$, and $h_{1}$ is linear in the intervals determined by the points $-1,-1+\varepsilon_{3}, 0, \varepsilon_{3}, 1$, then

$$
\int_{S^{2}} x_{3} \varphi_{h_{1}}(x) d \sigma(x)<0
$$


By (9), (10) there exists $s \in(0,1)$ such that if $h_{s}=s h_{0}+(1-s) h_{1}$ then

$$
\int_{S^{2}} x_{3} \varphi_{h_{s}}(x) d \sigma(x)=0 .
$$

Then $h_{s}:[-1,1] \rightarrow[-1,1]$ is continuous strictly increasing, $h_{s}(-1)=-1$, and $h_{s}(1)=1$. Hence by the properties of the $C_{t}$ 's, $-1 \leq t \leq 1$, it follows that there exists a homeomorphism $\varphi: S^{2} \rightarrow S^{2}$ such that if $\varphi=\left(\varphi_{1}, \varphi_{2}, \varphi_{3}\right)$ then $\varphi_{3}=\varphi_{h_{s}}$. By (8) and (11), it follows that

$$
\int_{S^{2}} x \varphi_{3}(x) d \sigma(x)=0 .
$$

Let $u: B^{3} \rightarrow B^{3}$ be the harmonic extension of $\varphi$. Then by (2) and (12) it follows that

$$
\nabla u_{3}(0)=0
$$

and hence

$$
\operatorname{det} J_{u}(0)=0 \text {. }
$$

Hence $u$ is not a diffeomorphism.

Remarks. 1. This example can be easily generalized to the $n$-dimensional case for $n \geq 3$. Also by being more careful in the construction of the $C_{t}$ 's and $h_{0}, h_{1}$, it is possible to construct a smooth diffeomorphism $\varphi: S^{2} \rightarrow S^{2}$ whose harmonic extension is not a diffeomorphism.

2. Actually we have constructed a homeomorphism $\varphi: S^{2} \rightarrow S^{2}$ whose third coordinate $\varphi_{3}: S^{2} \rightarrow[-1,1]$ is orthogonal to the coordinate functions $x_{1}, x_{2}, x_{3}$.

\section{REFERENCES}

[C] G. Choquet, Sur un type de transformation analytique généralisant la représentation conforme et définie au moyen de fonctions harmoniques, Bull. Sci. Math. (2) 69 (1945), 156-165.

[K] H. Kneser, Lösung der Aubgabe 41, Jber. Deutsch. Math.-Verein. 35 (1926), 123-124.

[R] T. Radó, Augabe 41, Jber. Deutsch. Math.-Verein. 35 (1926), 49.

Department of Mathematics, University of California at los Angeles, Los Angeles, CALIFORNIA 90024-1555 